\title{
Modelling indoor pollutant distribution via passive scalar and virtual box approach
}

\author{
George Pitchurov ${ }^{1, *}$, Detelin Markov ${ }^{1}$, Iskra Simova ${ }^{1}$, Rositsa Velichkova ${ }^{1}$, Peter Stankov ${ }^{1}$, \\ Radostina Angelova ${ }^{1}$ \\ ${ }^{1}$ Dept. PEPM, Technical University of Sofia, Bulgaria
}

\begin{abstract}
The distribution of PM2.5 around a thermal manikin with realistic female body shape in a naturally ventilated room has been modelled. The health risk (HR) due to inhalation of the PM2.5 has been quantified by integrating the pollutants mass flux over the boundaries of a virtual box around the mannequin's head (the breathing zone). By the same approach HR is evaluated over the boundaries of another virtual box that surrounds the manikins body and defines the occupied zone. The paper focuses on the peculiarities of creating and meshing a virtual geometry, as well as on the application of user-defined functions (UDF) for defining a pollutant source within the room using Ansys Fluent modelling package.
\end{abstract}

\section{Introduction}

PM2.5 readings are often included in air quality reports from environmental authorities and companies [1]. PM2.5 refers to atmospheric particulate matter (PM) that has a diameter of less than 2.5 micrometres. The size of those particles makes them susceptible to easy inhalation into the lungs, where they can inflict damage. It is also possible that these particles agglomerate viruses or bacteria and transport them into the human body. According to WHO [2], the well-known coronavirus (SARS-CoV-2) can also adhere to aerosols and be transmitted to lungs via inhalation. This transport mechanism is also addressed in [3-6]. To model the pollutant distribution in the room the passive-scalar model is used, since particles are too tiny to impact the flow field and tend to spread like a cloud. In such case the Eulerian and Lagrangian methods offer similar accuracy in predicting the contaminant concentration using RANS equations [7].

To quantify the risk of a human subject inhaling a PM2.5 pollutant, a virtual box approach was developed, where the subject head is surrounded by a box and the pollutants mass flux flowing through the box surfaces is calculated. Although it is possible to quantify surface fluxes in the post-processing level, the more robust solution is to create the box at the pre-processing level, i.e. during geometry creation. This required creation and manipulation of so-called virtual geometry in the mesh generator. This paper presents the steady-state emission and inhalation of pollutant PM2.5. To generate the pollutant in the room a user-defined function (UDF) approach was used, which allows the pollutant to be

*Corresponding author: george@tu-sofia.bg 
generated at arbitrary point in the domain without the need to construct a dedicated pollutant source inlet during the geometry creation stage.

\section{Problem formulation}

\subsection{Geometry and computational mesh}

A plan view of the simulated space is presented on Fig. 1. The room dimensions are $4 \mathrm{~m}$ length, $2.5 \mathrm{~m}$ width and $2.6 \mathrm{~m}$ height. The room has a window on the external wall and a closed door with a slot underneath on the opposite wall. There is a heater below the window and a table behind which a seated mannequin reclines. The mannequin numerical model is created through a topographic scanning of a real mannequin body by [8] and has been successfully imported into a numerical simulation for example in [9]. The mannequin has been scanned in a stereolitographic format and then converted to three-dimensional primitives in ACIS format before being imported into the mesh generator. The mannequin together with the location and dimensions of the virtual box around the head (the head box) are shown on Fig. 2. A second virtual box is constructed around the body of the occupant (body box) to identify a so-called occupant zone.

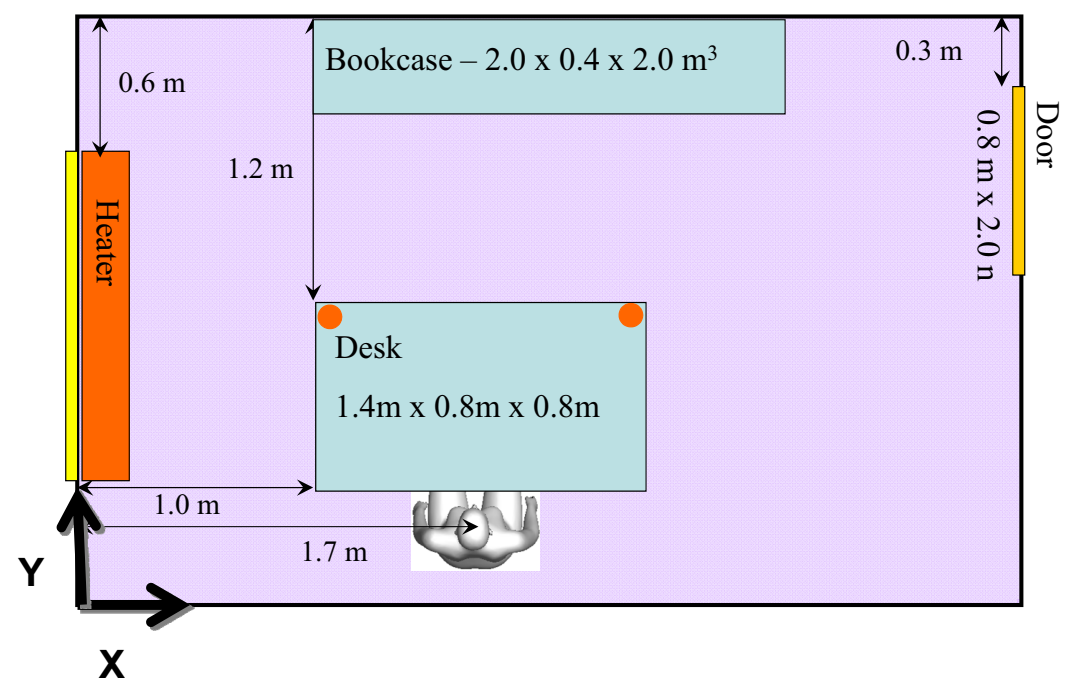

Fig. 1. Modeled enclosure with thermal mannequin.

The mesh was prepared with the Gambit mesh generator and consisted of 800000 tetrahedral cells (tets). This choice of cell type was mainly dictated by the complicated geometry near the mannekin body. Tets also allow for gradual coarsening of the mesh away from the body and are practically the only way to accomplish refinement while preserving the mesh conformal. Upon import in the mesh generator, the head of the mannequin is split by the surfaces of the head box. The body surface is meshed with triangular elements and the nostrils are selected to define an outlet (Fig. 3). Even though the body is well represented by the mesh, local refinement was attempted based on constraining the angle between unit normal vectors of neighbouring mesh cells. This procedure however didn't prove successful in $3 \mathrm{D}$ case as much as in $2 \mathrm{D}$, so it was abandoned. 

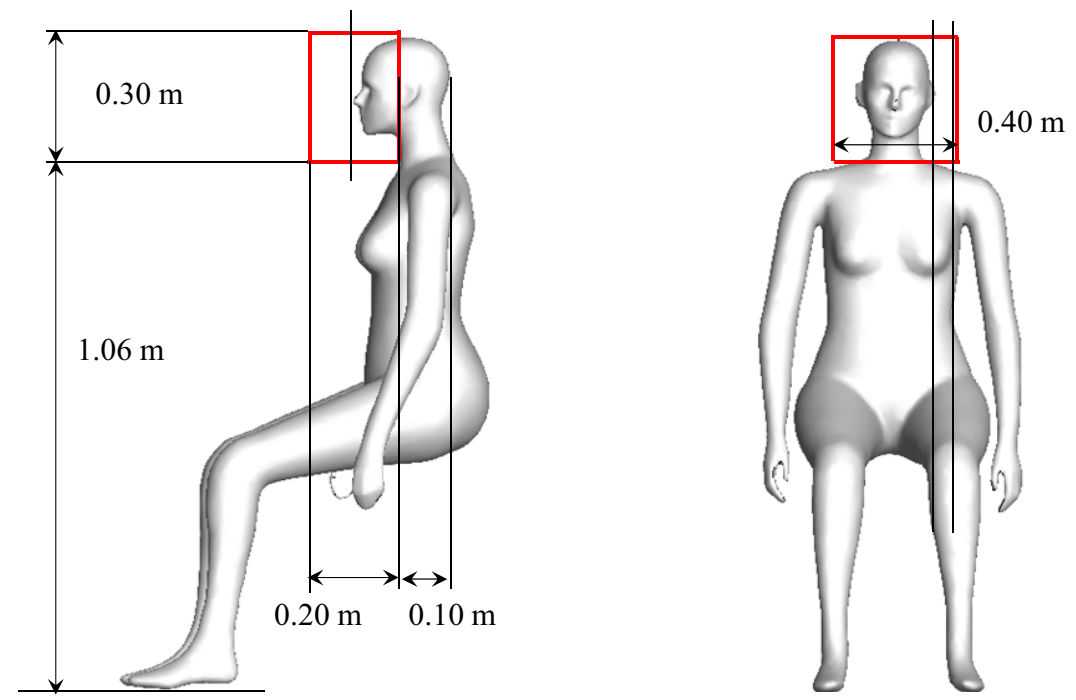

Fig. 2. Digitalized mannequin and the head box dimensions
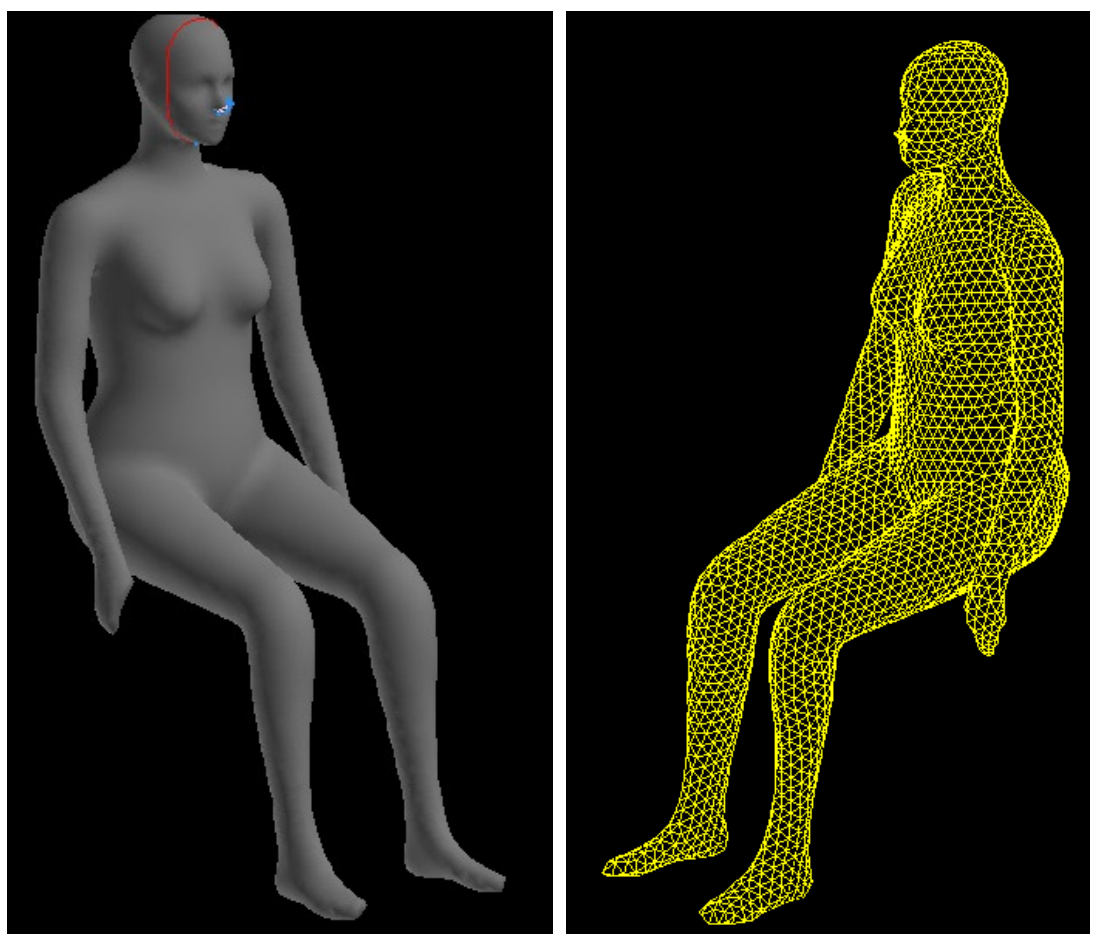

Fig. 3. Geometry and mesh of the mannequin body 


\subsection{Computational details}

The computations were done with Ansys 16.2 version of the Fluent software package in parallel mode on up to 4 computational nodes. For modelling the mean effect of turbulence the realizable version of the k-e model with enhanced wall treatment was used. The enhanced wall treatment models the wall effects by switching to low-Reynolds number model when the dimensionless wall distance $\mathrm{y}+$ is lower than 5 , and adopts wall function approach when $\mathrm{y}^{+}>30$. In the region between these two limits a blending technique is employed. For modelling of the thermal effects on the flow the Boussinesq approach was used. The fluid is treated as incompressible, but allowing for buoyancy effects by introducing in the Z-momentum equation a source term proportional to a temperature difference and the thermal expansion coefficient of air. The Boussinesq approach is applicable when temperature difference between inlet and outlet are much lower in magnitude compared to the absolute temperature, which in the simulated cases was less than $1 \%$. The radiative heat transfer was excluded from the simulation at this stage since it was assumed that the temperature difference were rather small for the radiative heat transfer to significantly change the velocity field that governs the pollutant transfer.

The SIMPLE pressure-velocity coupling was employed since fluid is incompressible (with respect to pressure). Momentum variables were discretized with a second-order upwind scheme and the scalar and temperature variables with a first-order. The first-order scheme has the property to be more conservative and to suppress solution oscillations beyond the physical limits of the variable.

The passive-scalar model is suitable for low pollutant concentrations, as discussed, however a proper treatment of the turbulent scalar diffusion is necessary, which has been ensured with the help of a user-defined function. For correct conservation of the pollutant it is also important to disable the scalar diffusion through the inlet. This is necessary since part of the scalar will leave the domain through the inlet via diffusion and the resulting mass flux at the outlet will not be equal to the one generated at the source. Pollutant distribution in the investigated space was calculated as a second step in a so-called frozen velocity field, after the flow field (velocity and temperature) has been converged.

\subsection{Boundary conditions}

Outdoor air enters through the whole window with a velocity of $0.01 \mathrm{~m} / \mathrm{s}$ which corresponds to a volume flow rate of $0.0112 \mathrm{~m}^{3} / \mathrm{s}$. The domain has two outlets: the first one is at the slot below the door and the second one is at the mannequin's nostrils. The door slot was extruded $1 \mathrm{~m}$ further out of the domain to ensure fully developed flow and avoid recirculation. The door slot outlet pressure was fixed to zero, while the occupant's nostrils were assigned target mass flow rate of $0.12 .10^{-3} \mathrm{~kg} / \mathrm{s}$, which is roughly $1 / 100$ of the room inflow. The boundary condition at the nostrils is still a static pressure, but it evolves dynamically based on the required mass flow rate.

For boundary conditions of pollutant a zero value was used on inlets. For the outlets a zero gradient (which corresponds to zero diffusive flux) was used. The zero gradient still allows a flow of pollutant, but assumes a fully developed flow. This is true for the slot outlet, but not so much for the mannequin nostrils, unless a deeper extrusion into the mannequin head is attempted. This wasn't done here because once the pollutant flux at the door slot is determined correctly, the mannequin nostrils flux will emerge naturally from mass conservation when the simulation converges.

To allow for proper mass conservation of pollutant, the inlet diffusion was disabled due to reasons discussed above, i.e. to disallow the flow of pollutant through the inlet. If the diffusion is not disabled, any non-zero gradient in the vicinity of the inlet will create flux 
due to Fick's law and will let some pollutant to escape. This will create discrepancy between the pollutant flow rate at the outlets and the generation rate. In the rest of the domain the pollutant was of course allowed to diffuse. The diffusion coefficient of the pollutant was hard-linked to the turbulent momentum diffusion with a turbulent Prandtl number of 0.9 . This modification is crucial for the realistic prediction of pollutant diffusion via turbulence.

Five pollutants were simultaneously simulated, three of them generated over the desk and two generated over the bookcase. The pollutant source coordinates are presented in Table 1. Source locations were assigned via a user-defined function (UDF), which scanned the numerical grid to find the cell closest to the pre-defined source location (appendix A). These cells were then assigned a pollutant source with a strength of $1 \mathrm{E}-09 \mathrm{~kg} / \mathrm{s}$. The advantage of using UDF instead of hard-fixing the location was the flexibility to change the pollutant location without modifying the mesh.

Table 1 Diffusive sources of pollutant PM2.5

\begin{tabular}{|c|c|c|c|c|}
\hline \multirow{2}{*}{ Pollutant } & \multicolumn{3}{|c|}{ Coordinates [m] } & \multirow{2}{*}{ Location } \\
\hline & $\mathbf{X}$ & $\mathbf{Y}$ & $\mathbf{Z}$ & \\
\hline UDS0 & 1.6 & 1.2 & 0.85 & Desk left \\
\hline UDS1 & 2.0 & 1.31 & 0.85 & Desk middle \\
\hline UDS2 & 2.4 & 1.2 & 0.85 & Desk right \\
\hline UDS3 & 1.1 & 2.3 & 2.05 & Bookcase \\
\hline UDS4 & 2.9 & 2.3 & 2.05 & Bookcase \\
\hline
\end{tabular}

Regarding thermal boundary conditions the mannequin body surface was assigned a heat flux of $70 \mathrm{~W} / \mathrm{m}^{2}$. The heat output from heater was $320 \mathrm{~W}$, which was split between its front and top surfaces. The inflow air temperature through the window was fixed at $296 \mathrm{~K}$. All walls (incl. floor and ceiling) were adiabatic except for the one that separates the room from the outdoors, on which the window is installed. This wall was assigned a heat loss of $100 \mathrm{~W} / \mathrm{m}^{2}$.

\section{Results and discussion}

\subsection{Velocity and temperature}

Richardson number for the flow was on the order of $10^{3}$, which indicates a much higher impact of natural compared to forced convection. The numerical results confirmed that the flow is dominated by the thermal plumes from mannequin and heater and not so much from the low-momentum flow through the window. Fig. 4 shows the temperature field in a plane cut through the mannequin, where the thermal plume is clearly visible. 

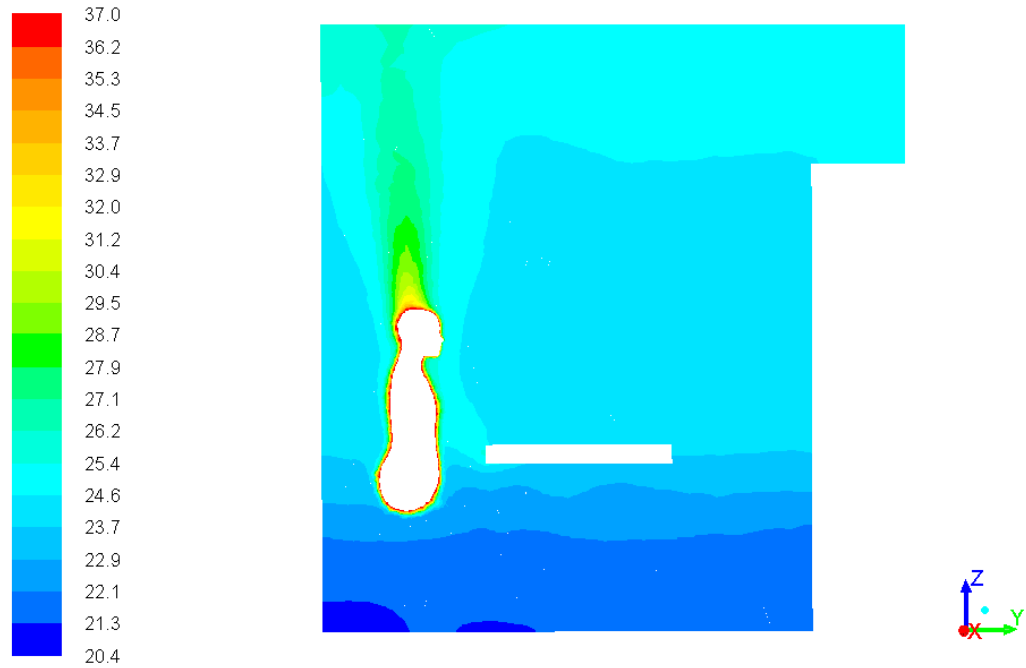

Fig. 4. Temperature contours $\left[{ }^{\circ} \mathrm{C}\right]$ in a mid-plane through the mannequin.

The resulting natural convection as predicted by the Boussinesq model is visualized by the velocity vector field in Fig. 5. Similar is the convective plume above the heater. This natural boundary layer cannot be penetrated by external airflows in the studied setup. This has the consequence that this layer can protect the person from contamination transported by external flows, which is the underlying principle of displacement ventilation [10]. However, if a contaminant source is located at the beginning of the boundary layer then the layer will entrain it and will transport the contaminant to the breathing zone.

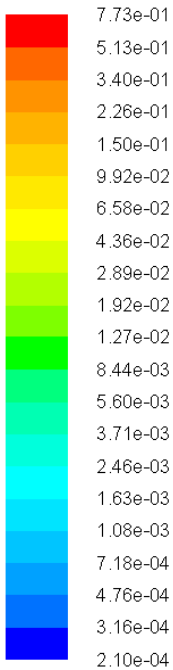

Fig. 5. Velocity vectors in a mid-plane through the mannequin. 


\subsection{Contaminants}

The mid-plane view from the previous section is used in Fig. 6 through Fig. 10 to show the distribution of contaminants for the five sources listed in Table 1. In the absence of strong flows near the contaminant source the contaminant is transported by turbulent diffusion, as can be seen for example in UDS0 in Fig. 6. The contaminant is further entrained by the thermal convective plume created by the heated human body and transported most likely to the breathing zone. To determine the pollutant concentration in the breathing zone the head box pollutant concentration is needed. It is visualized in Fig. 11 through Fig. 15 for the five simulated contaminants.
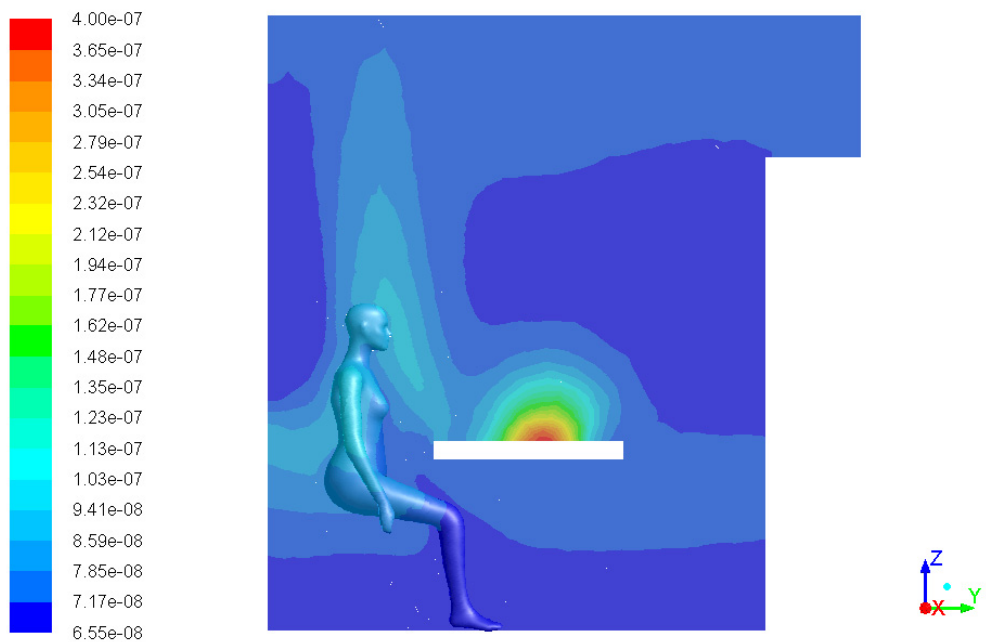

Fig. 6. Mass fraction of pollutant UDS0 $[\mathrm{kg} / \mathrm{kg}]$ in a vertical mid-plane through the mannequin
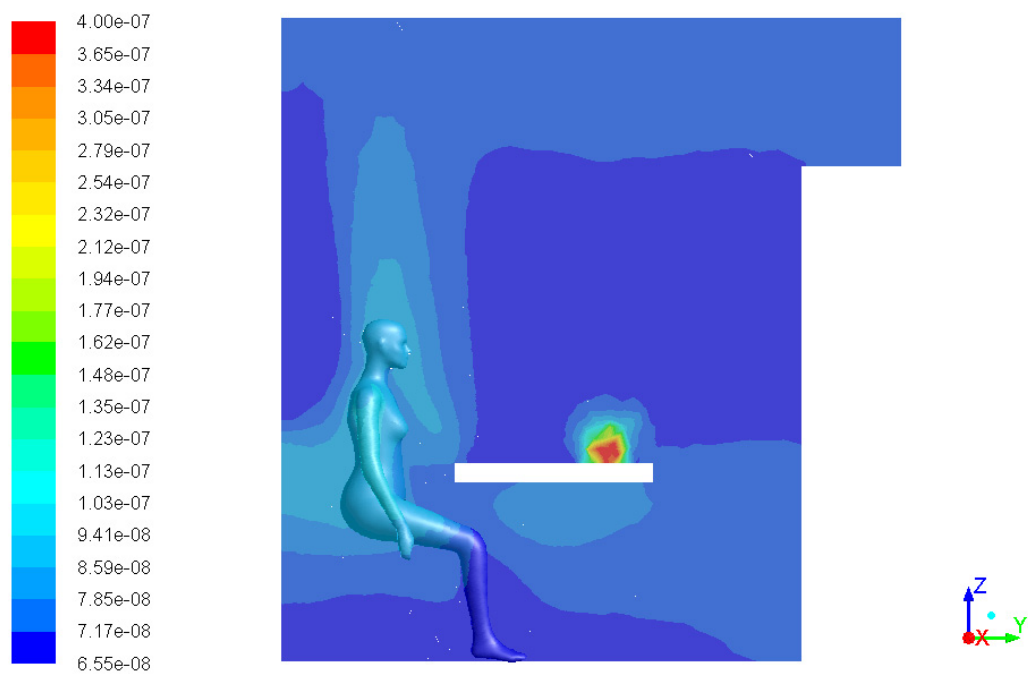

Fig. 7. Mass fraction of pollutant UDS $1[\mathrm{~kg} / \mathrm{kg}]$ in a vertical mid-plane through the mannequin 

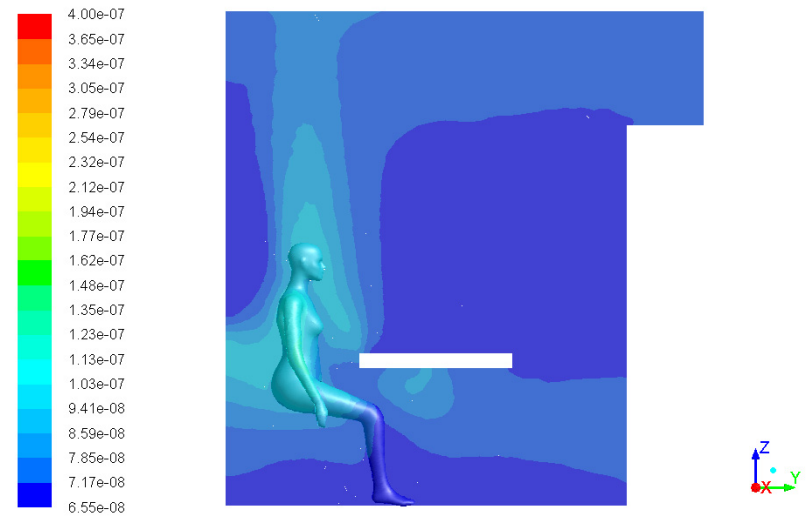

Fig. 8. Mass fraction of pollutant UDS $2[\mathrm{~kg} / \mathrm{kg}]$ in a vertical mid-plane through the mannequin
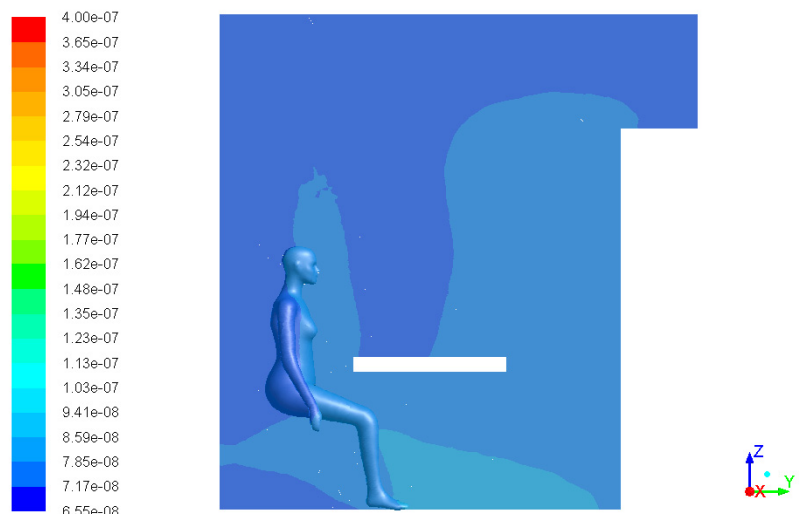

Fig. 9. Mass fraction of pollutant UDS3 $[\mathrm{kg} / \mathrm{kg}]$ in a vertical mid-plane through the mannequin
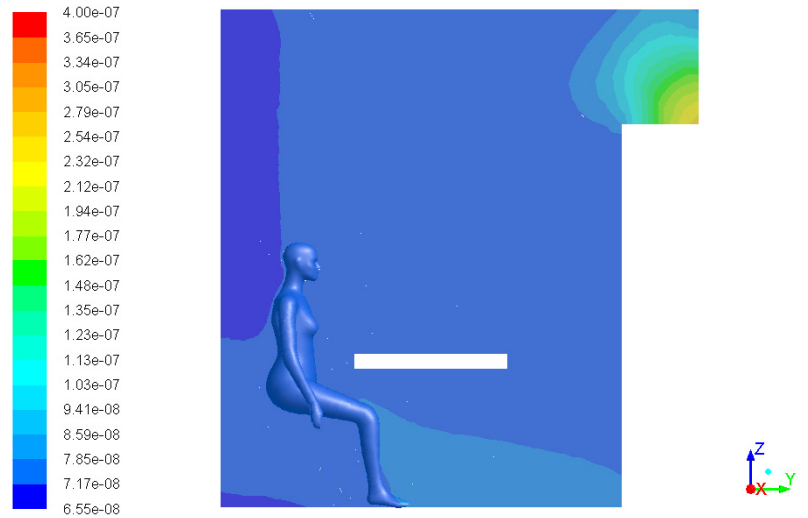

Fig. 10. Mass fraction of pollutant UDS4 $[\mathrm{kg} / \mathrm{kg}]$ in a vertical mid-plane through the mannequin 

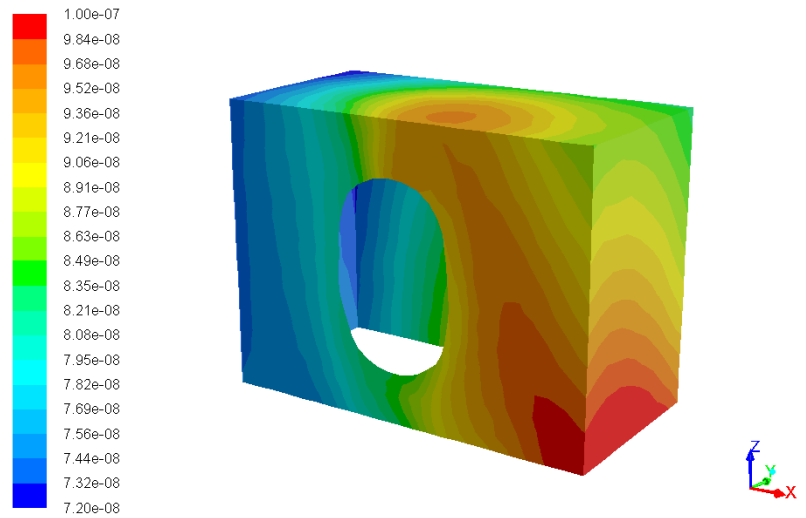

Fig. 11. Mass fraction of pollutant UDS0 $[\mathrm{kg} / \mathrm{kg}]$ over the virtual mannequin head-box.
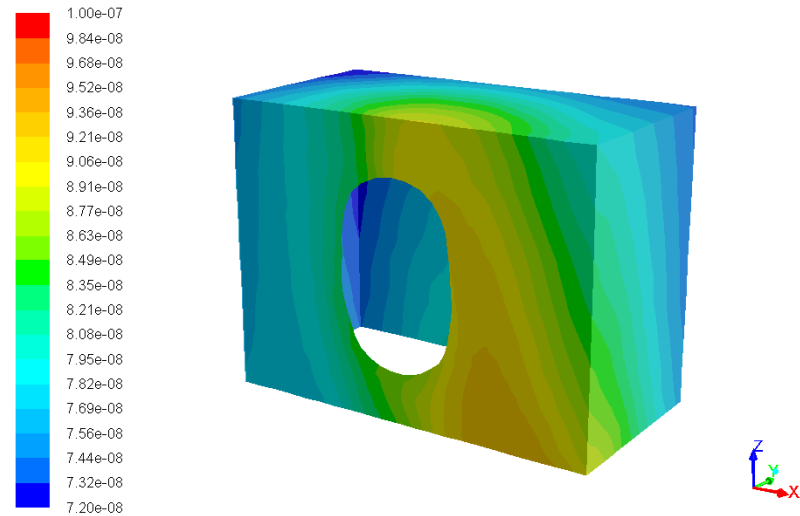

Fig. 12. Mass fraction of pollutant UDS1 $[\mathrm{kg} / \mathrm{kg}]$ over the virtual mannequin head-box.
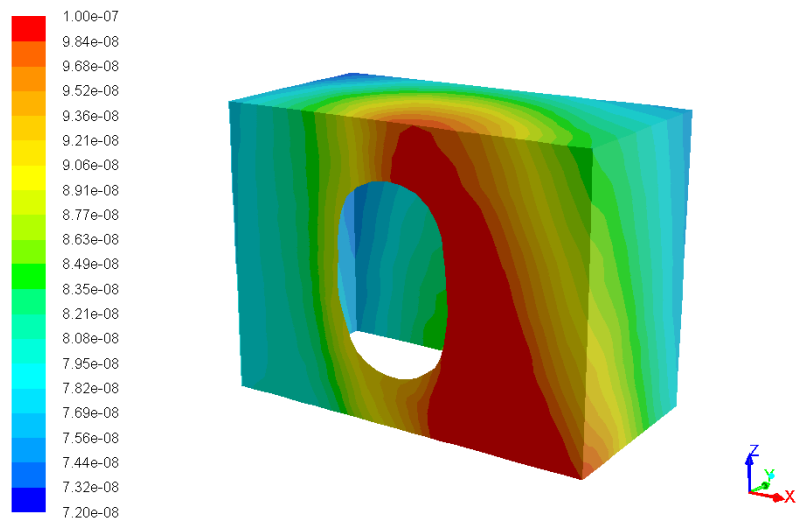

Fig. 13. Mass fraction of pollutant UDS2 [kg/kg] over the virtual mannequin head-box. 


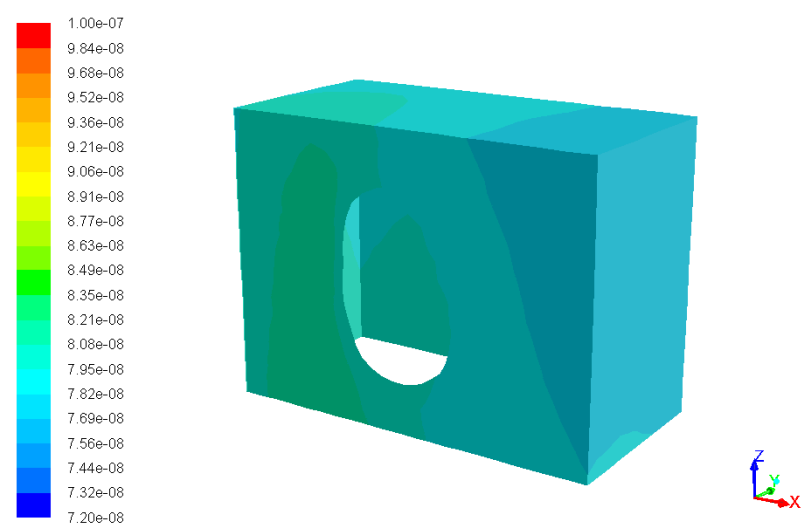

Fig. 14. Mass fraction of pollutant UDS3 [kg/kg] over the virtual mannequin head-box.
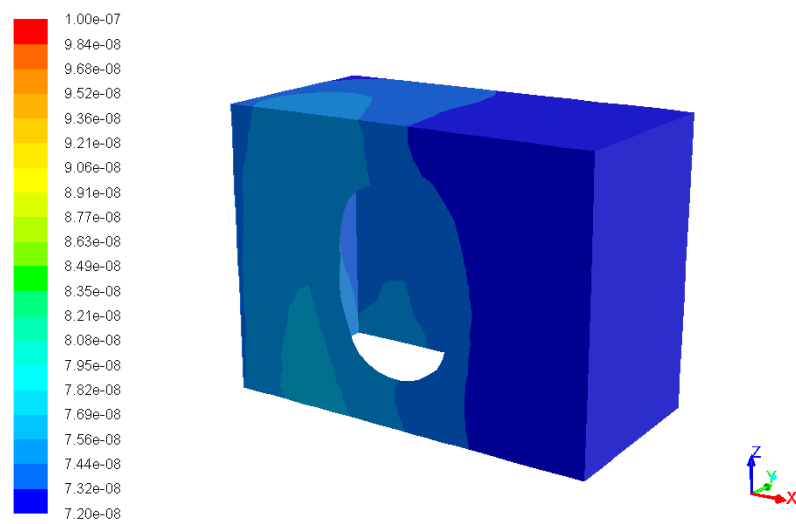

Fig. 15. Mass fraction of pollutant UDS4 [kg/kg] over the virtual mannequin head-box.
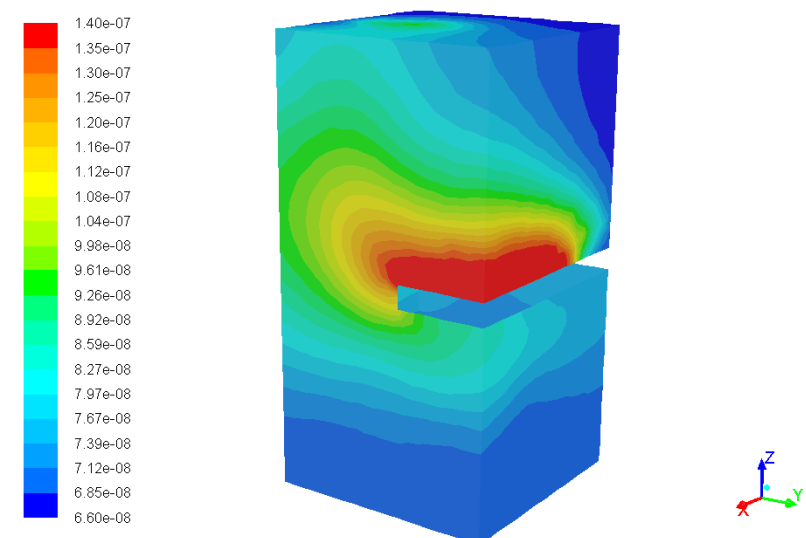

Fig. 16. Mass fraction of pollutant UDS $0[\mathrm{~kg} / \mathrm{kg}]$ over the virtual body-box 
Fig. 16 through Fig. 20 depict the mass fraction of pollutant over the surface of the virtual body box. What is to be noted is the higher gradient of pollutant when the latter is located on the desk, i.e. close to the considered virtual surface. Being close to the surface prohibits the contaminant from mixing before entering the body box. On the other hand contaminants located further have sufficient time and space to mix and dilute. As a result the contaminants generated from the bookcase offer much uniform concentration over the body-box surface. The overall quantity of contaminant entering the body box however is rather equal between contaminants and independent on the location, which will become clear in a later analysis.
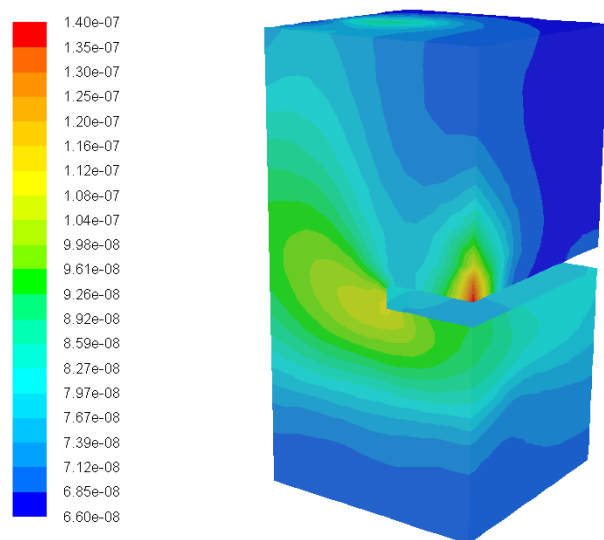

Fig. 17. Mass fraction of pollutant UDS1 $[\mathrm{kg} / \mathrm{kg}]$ over the virtual body-box
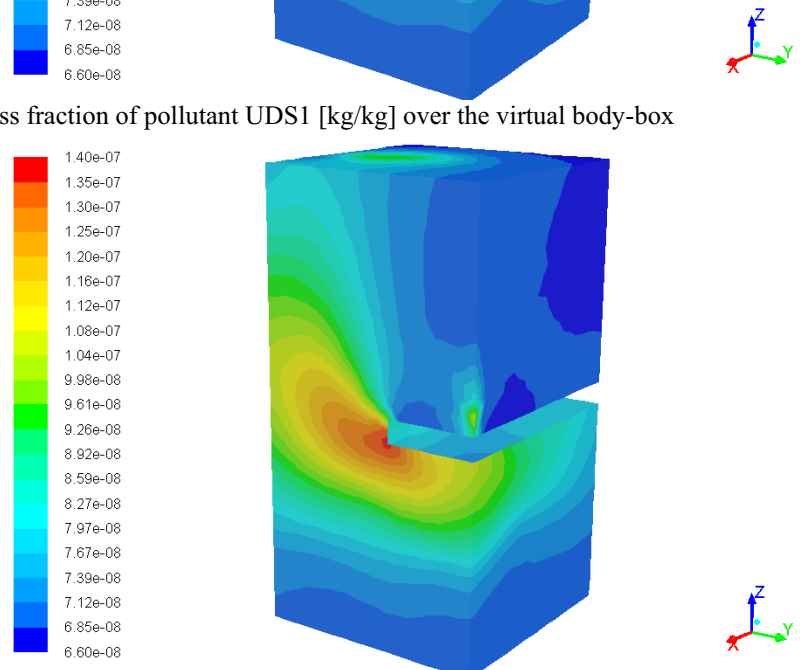

$1.35 \mathrm{e}-07$
$1.30 \mathrm{e}-07$
$1.25 \mathrm{e}-07$
$1.20 \mathrm{e}-07$
$1.16 \mathrm{e}-07$
$1.12 \mathrm{e}-07$
$1.08 \mathrm{e}-07$
$1.04 \mathrm{e}-07$
$9.98 \mathrm{e}-08$
$9.61 \mathrm{e}-08$
$9.26 \mathrm{e}-08$
$8.92 \mathrm{e}-08$
$8.59 \mathrm{e}-08$
$8.27 \mathrm{e}-08$
$7.97 \mathrm{e}-08$
$7.67 \mathrm{e}-08$
$7.39 \mathrm{e}-08$
$7.12 \mathrm{e}-08$
$6.85 \mathrm{e}-08$
$6.60 \mathrm{e}-08$

Fig. 18. Mass fraction of pollutant UDS $[\mathrm{kg} / \mathrm{kg}]$ over the virtual body-box

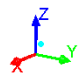



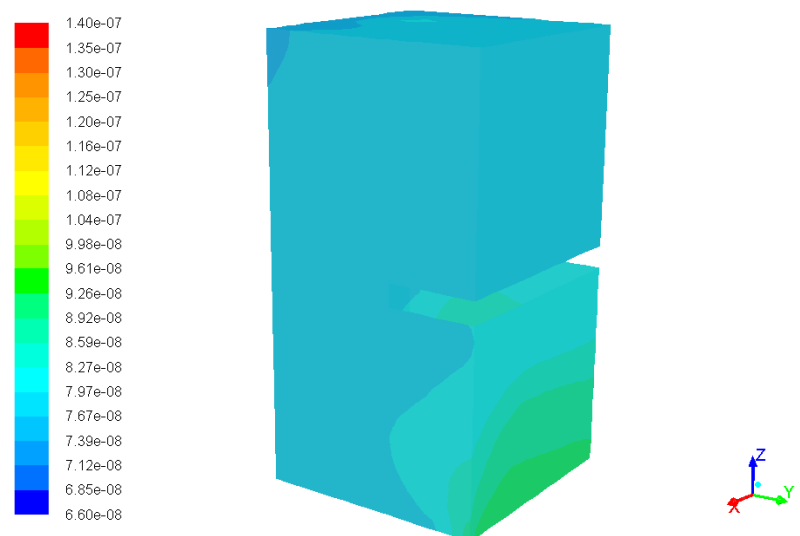

Fig. 19. Mass fraction of pollutant UDS $3[\mathrm{~kg} / \mathrm{kg}]$ over the virtual body-box
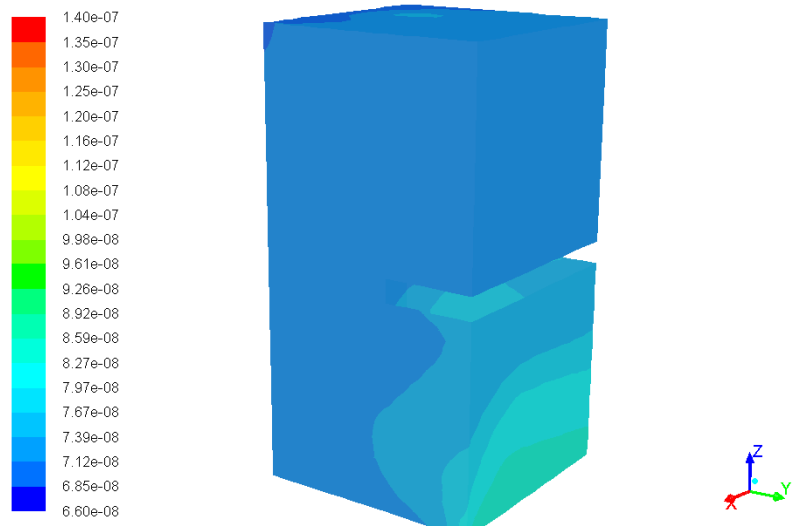

Fig. 20. Mass fraction of pollutant UDS4 $[\mathrm{kg} / \mathrm{kg}]$ over the virtual body-box

Concentration alone can not determine uniquely the inhaled pollutant quantity as the latter is an integral of the concentration by the air mass flowrate over the box surface. That means zones with high concentration may not lead to higher inhalation if not enough air flows through them and vice versa-a small segment with high concentration may increase the inhaled quantity significantly if enough air is transported through it. Table 2 presents the net flow rate of the five pollutants through the surface of both boxes (the head and body box), as well as the flowrate and mass fraction over the mannequin nose during inhalation mode. The table also presents normalized quantities obtained by dividing the corresponding flow rates by the source strength $\left(1.10^{-9} \mathrm{~kg} / \mathrm{s}\right)$. In this way it becomes visible what fraction of the emmited contaminant actually enters the occupied and the breathing zone. It should be noted that the flow rate is calculated as an integral of the velocity over the surface area, i.e. it represents the convective flux of the pollutatnt through the area. However, concentration gradients in the viccinity will also contribute to a diffusive flux, which is not accounted for in this calculus due to software limitations. Although the door outlet was extruded to provide for zero diffusive flux, any internal surface of the domain, over which integral balance is calculated, will also be subject to a diffusive flux, which is not accounted 
for. This will explain certain discrepancy observed between the flow rates through the boxes compared to inhaled flow rates.

The inhaled fraction of contaminant is calculated by dividing the contaminant flowrate through the nose by the emission rate from the source. The result is depicted on Fig. 21 as a percentage, showing that roughly $1 \%$ from the generated contaminant is inhaled by the person. It is seen that contaminant sources closer to the occupant generally result in higher inhaled fraction. The observed difference between inhaled fractions is however not so large and is not proportional to the proximity of the pollutant source. The likely reason is that turbulent mixing in the room would homogenize the pollutant and thus smear out the difference between the different pollutant setups. Fig. 22 depicts the turbulent diffusion coefficient of pollutant in a room mid-plane. The coefficient is calculated from the turbulent viscosity and a turbulent Prandtl number of 0.9 for all contaminants alike. The figure shows high value of diffusion coefficient in the majority of the room and thus a strong mixing augmented by turbulence. This will reduce the difference between the different pollutant flowrates at the sampled surfaces.

Table 2 Flowrates and mass fraction of pollutant PM2.5

\begin{tabular}{|c|c|c|c|c|c|}
\hline & UDS0 & UDS1 & UDS2 & UDS3 & UDS4 \\
\hline Flowrate headbox $[\mathrm{kg} / \mathrm{s}]$ & $4.52 \mathrm{E}-11$ & $3.10 \mathrm{E}-11$ & $3.97 \mathrm{E}-11$ & $1.66 \mathrm{E}-11$ & $1.54 \mathrm{E}-11$ \\
\hline $\begin{array}{l}\text { Normalized flowrate } \\
\text { headbox [-] }\end{array}$ & 4.52E-02 & $3.10 \mathrm{E}-02$ & $3.97 \mathrm{E}-02$ & $1.66 \mathrm{E}-02$ & $1.54 \mathrm{E}-02$ \\
\hline Flowrate bodybox $[\mathrm{kg} / \mathrm{s}]$ & $1.75 \mathrm{E}-10$ & $9.67 \mathrm{E}-11$ & $5.08 \mathrm{E}-11$ & $9.30 \mathrm{E}-11$ & $8.64 \mathrm{E}-11$ \\
\hline $\begin{array}{l}\text { Normalized flowrate } \\
\text { bodybox [-] }\end{array}$ & $1.75 \mathrm{E}-01$ & $9.67 \mathrm{E}-02$ & $5.08 \mathrm{E}-02$ & $9.30 \mathrm{E}-02$ & $8.64 \mathrm{E}-02$ \\
\hline Flowrate nose $[\mathrm{kg} / \mathrm{s}]$ & $1.29 \mathrm{E}-11$ & $1.24 \mathrm{E}-11$ & $1.36 \mathrm{E}-11$ & $1.11 \mathrm{E}-11$ & $1.03 \mathrm{E}-11$ \\
\hline $\begin{array}{l}\text { Normalized flowrate nose } \\
{[-]}\end{array}$ & $1.29 \mathrm{E}-02$ & $1.24 \mathrm{E}-02$ & $1.36 \mathrm{E}-02$ & $1.11 \mathrm{E}-02$ & $1.03 \mathrm{E}-02$ \\
\hline $\begin{array}{l}\text { Nose mass fraction } \\
{[\mathrm{kg} / \mathrm{kg}]}\end{array}$ & $9.20 \mathrm{E}-08$ & $8.84 \mathrm{E}-08$ & $9.73 \mathrm{E}-08$ & 7.92E-08 & 7.37E-08 \\
\hline
\end{tabular}

\section{Inhaled contaminant fraction}

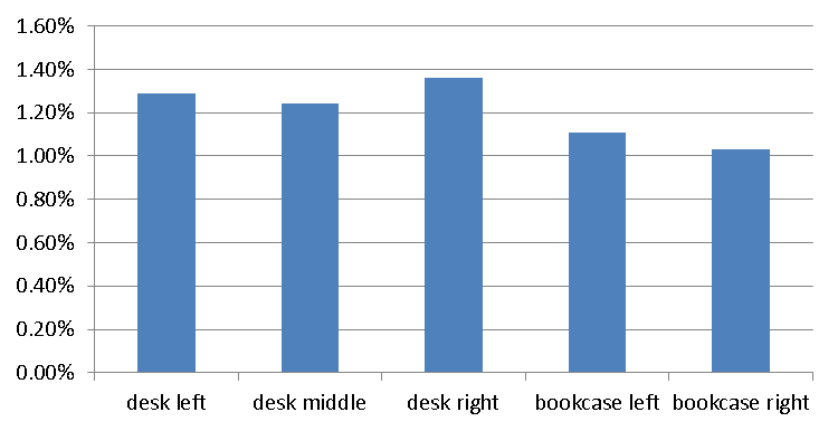

Fig. 21. Fraction of inhaled contaminant as a percentage from the contaminant emission rate in the room. 

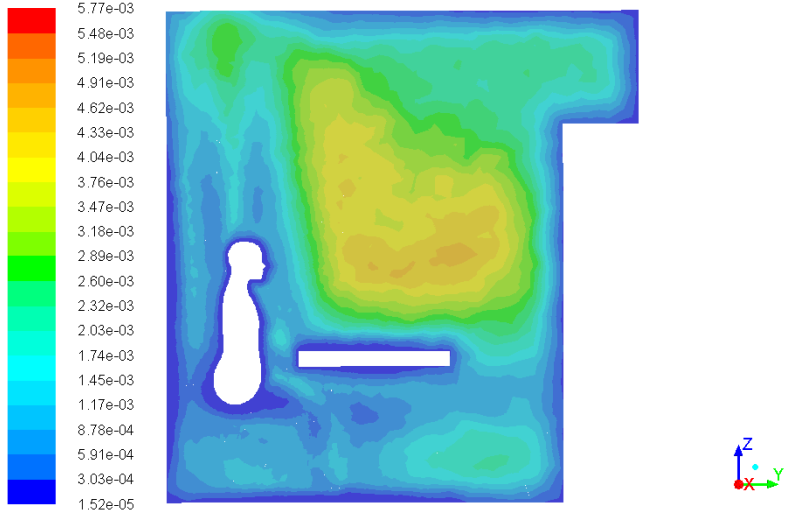

Fig. 22. Turbulent diffusion coefficient of contaminant $[\mathrm{kg} / \mathrm{m} . \mathrm{s}]$

\section{Conclusion}

Inhalation risk of PM2.5 was estimated by introducing 5 contaminants in a naturally ventilated room and calculating the percentage of contaminant inhaled by a thermal mannequin. Breathing zone and occupied zone have been analysed by introducing a head and body box as a virtual geometry in the simulation. Inhalation risk evaluated with the means of both the head box and the body box could be different than the inhalation risk calculated by the inhaled pollutant flux through the nostrils of the mannequin. Inhalation risk turned to be not much dependant on contaminant source distance, but this was due to the well mixed room airflow. The mixing was augmented by thermal plumes created by the heated mannequin body and a room heater. Concentration gradients, although not so big, impacted the accuracy of contaminant mass flow estimation through the virtual box surfaces, because gradients contribute to an extra diffusive mass flux. As a result, risk estimates were more credible when using the inhaled mass flow rate of contaminant. Because the room air was rather well mixed, the contaminant flow rate ratio was close to the airflow ratio between the mannequin nose and the room outlet, or roughly 1:100. In a poorly mixed case the result would likely be different, and thus the result will depend on both the flow field and contaminant location.

This work was supported by the European Regional Development Fund within the Operational Programme "Science and Education for Smart Growth 2014 - 2020" under the Project CoE "National center of mechatronics and clean technologies "BG05M2OP001-1.001-0008".

\section{APPENDIX A. User-defined function (UDF) for Ansys Fluent to generate a scalar contaminant at user-defined location in the room.}

\#include "udf.h"

\#define NUM_POINTS 5

real source $=1.0 \mathrm{e}-9 ; / /[\mathrm{kg} / \mathrm{s}]$ 


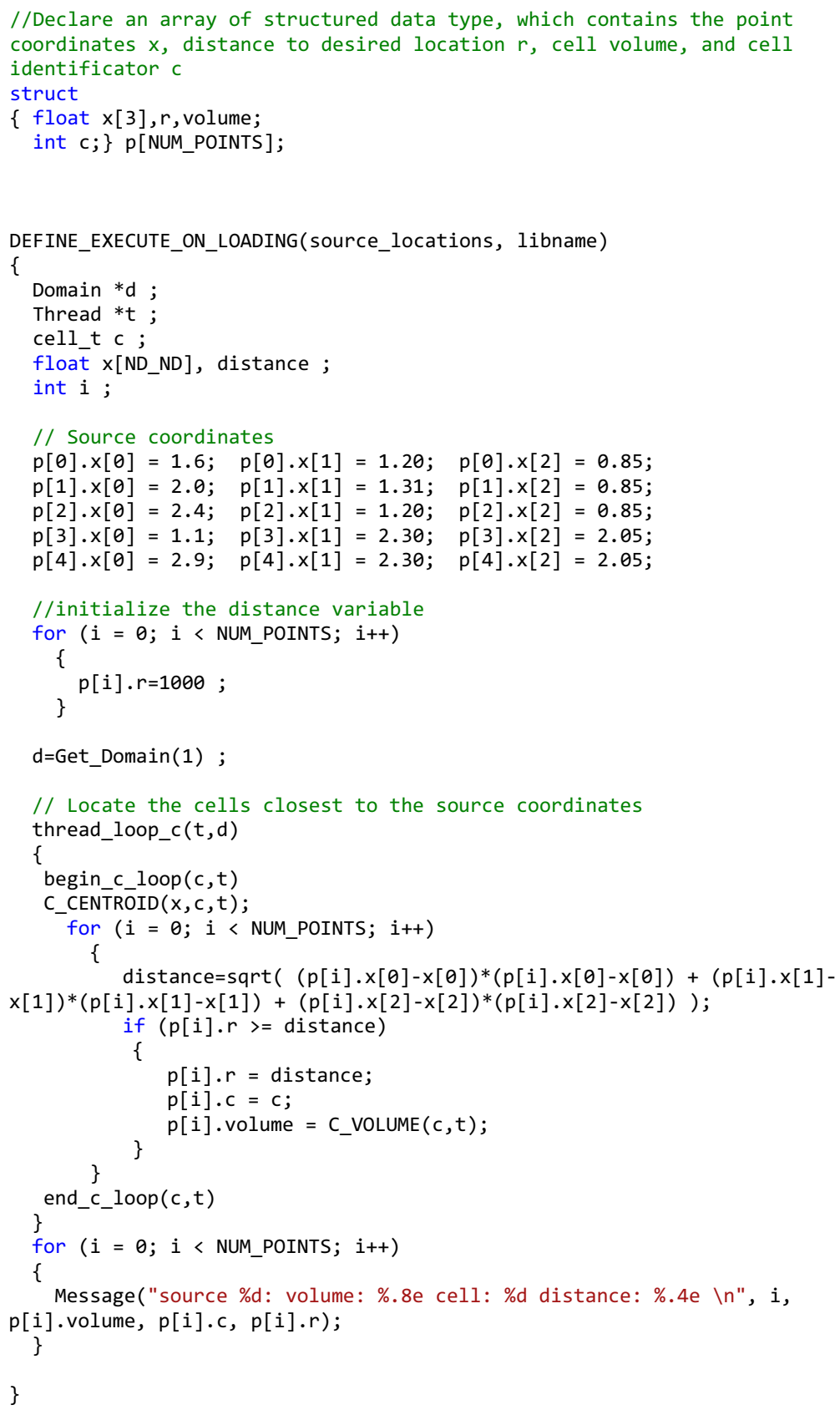




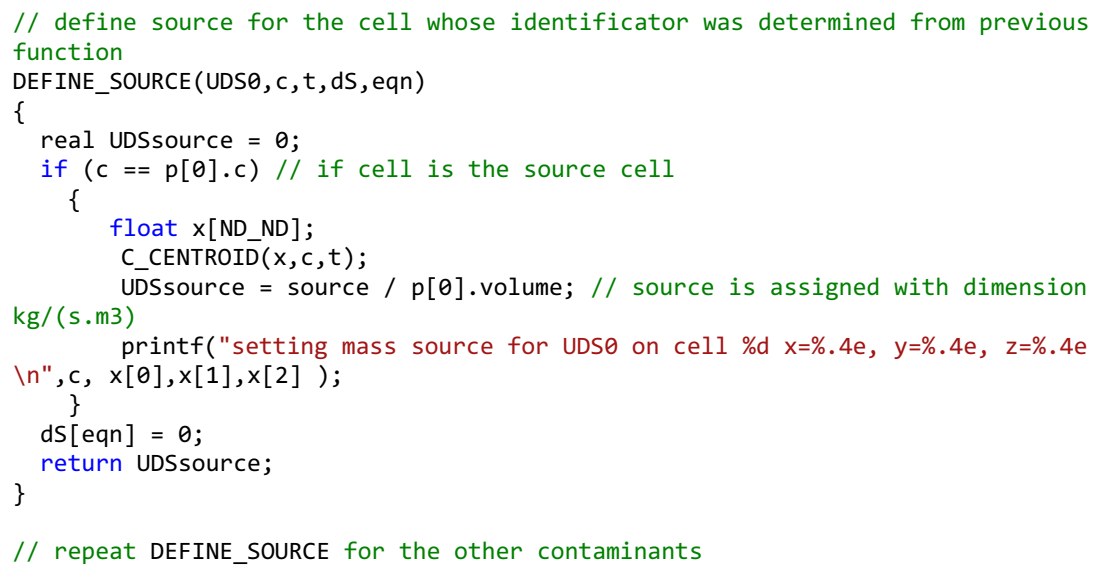

\section{References}

1. Bliss Air, https://blissair.com/what-is-pm-2-5.htm

2. World health organization https://www.who.int/emergencies/diseases/novelcoronavirus-2019/question-and-answers-hub/q-a-detail/coronavirus-disease-covid-19how-is-it-transmitted

3. Yu, Y. X., Sun, L., Yao, K., Lou, X. T., Liang, X., Zhao, B. W., ... \& Zhang, H. (2020). Consideration and prevention for the aerosol transmission of 2019 novel coronavirus. [Zhonghua yan ke za zhi] Chinese journal of ophthalmology, 56, E008E008.

4. Lv, J., Gao, J., Wu, B., Yao, M., Yang, Y., Chai, T., \& Li, N. (2021). Aerosol Transmission of Coronavirus and Influenza Virus of Animal Origin. Frontiers in Veterinary Science, 8, 109.

5. Azimi, P., Keshavarz, Z., Laurent, J. G. C., Stephens, B., \& Allen, J. G. (2021). Mechanistic transmission modeling of COVID-19 on the Diamond Princess cruise ship demonstrates the importance of aerosol transmission. Proceedings of the National Academy of Sciences, 118(8).

6. Amirav, I., \& Newhouse, M. T. (2020). Transmission of coronavirus by nebulizer: a serious, underappreciated risk. Cmaj, 192(13), E346-E346.

7. Zhang, Z. and Chen, Q. 2007. "Comparison of the Eulerian and Lagrangian methods for predicting particle transport in enclosed spaces," Atmospheric Environment, 41(25), 5236-5248.

8. Nielsen P. V., Murakami S., Kato S., Topp C., et al., Benchmark Tests for a Computer Simulated Person, ISSN 1395-7953 R0307, Department of Building, Technology and Structural Engineering, Aalborg University, 2003, http://cfd-benchmarks.com/

9. Pichurov G., P. Stankov, Integration of thermophysiological body model in CFD, Cent. Eur. J. Eng., 3(3), 2013, 513-521, DOI: 10.2478/s13531-012-0071-8

10. Xing, Huijuan, Andy Hatton, and Hazim B. Awbi. "A study of the air quality in the breathing zone in a room with displacement ventilation." Building and environment 36.7 (2001): 809-820. 